math.QA/0602077

KCL-MTH-06-02

Hamburger Beiträge zur Mathematik Nr. 231

ZMP-HH/05-30

\title{
Twining characters and Picard groups in rational conformal field theory
}

\author{
Christoph Schweigert, Jürgen Fuchs, and Ingo Runkel
}

\begin{abstract}
Picard groups of tensor categories play an important role in rational conformal field theory. The Picard group of the representation category $\mathcal{C}$ of a rational vertex algebra can be used to construct examples of (symmetric special) Frobenius algebras in $\mathcal{C}$. Such an algebra $A$ encodes all data needed to ensure the existence of correlators of a local conformal field theory.

The Picard group of the category of $A$-bimodules has a physical interpretation, too: it describes internal symmetries of the conformal field theory, and allows one to identify generalized Kramers-Wannier dualities of the theory.

When applying these general results to concrete models based on affine Lie algebras, a detailed knowledge of certain representations of the modular group is needed. We discuss a conjecture that relates these representations to those furnished by twining characters of affine Lie algebras.
\end{abstract}

\section{Modular tensor categories and conformal blocks}

Vertex algebras constitute a concise formalization of the heuristic concept of a chiral symmetry algebra in conformal field theory. They are, by now, well established as a mathematical structure, and have applications in various mathematical disciplines. One aspect that makes their theory particularly rich is the existence of a notion of tensor product for modules over a vertex algebra. The theory of such tensor products was developed in a series of articles HL1, HL2, HL3, HL4, Hu1 which form one of Jim Lepowsky's important contributions to the field. It closely links vertex algebras to tensor categories.

In recent work $\mathbf{H u 2}$, Huang extends this relationship by identifying a class of vertex algebras for which the representation category carries the stronger structure of a modular tensor category. This justifies the point of view, initiated in the work of Moore and Seiberg [MS], that modular tensor categories furnish an axiomatization of the chiral data of rational two-dimensional conformal quantum field theory.

For the present contribution, we adopt the following definition of a modular tensor category: it is an abelian, semi-simple, $\mathbb{C}$-linear monoidal category $\mathcal{C}$, equipped

2000 Mathematics Subject Classification. 81T40,18D10,18D35,81T45.

Key words and phrases. Rational conformal field theory, modular tensor categories, topological field theory, vertex algebras.

C.S. is supported by the DFG project SCHW 1162/1-1, and J.F. by VR under project no. $621-2003-2385$. 
with a functorial braiding $c_{X, Y}: X \otimes Y \stackrel{\sim}{\rightarrow} Y \otimes X$ and functorial twist isomorphisms $\theta_{X}: X \stackrel{\sim}{\rightarrow} X$, subject to the following axioms:

- Braiding and twist are compatible in the sense that $\theta_{X \otimes Y}=c_{Y, X} \circ\left(\theta_{Y} \otimes \theta_{X}\right) \circ c_{X, Y}$.

- There exists a compatible duality $X \mapsto X^{\vee}$ on $\mathcal{C}$.

- The tensor unit $\mathbf{1}$ is simple.

- There are only finitely many isomorphism classes of simple objects.

We denote a set of representatives for these classes by $\left\{U_{i}\right\}_{i \in I}$.

- Finally, there is a non-degeneracy condition on the braiding.

It ensures in particular that the natural transformations of the identity functor on $\mathcal{C}$ are controlled by the fusion $\operatorname{ring} K_{0}(\mathcal{C})$, in the sense that

$$
\operatorname{End}\left(\operatorname{Id}_{\mathcal{C}}\right) \cong K_{0}(\mathcal{C}) \otimes_{\mathbb{Z}} \mathbb{C} \text {. }
$$

By results of Reshetikhin and Turaev $\mathbf{R T}$, to each modular tensor category one can associate a monoidal category $\mathrm{Cob}_{\mathcal{C}}$ of decorated cobordisms and a monoidal functor $\mathrm{tft}_{\mathcal{C}}$ from $\mathrm{Cob}_{\mathcal{C}}$ (or rather, a version of this category that is enriched over $\left.\left.\mathcal{V}_{e c t}\right)_{\mathbb{C}}\right)$ into the category $\mathcal{V}_{e c t}$ of finite-dimensional complex vector spaces. The objects of $\mathrm{Cob}_{\mathcal{C}}$ are called extended surfaces; an extended surface E is an oriented closed two-dimensional topological manifold, together with a Lagrangian subspace of $H_{1}(\mathrm{E}, \mathbb{R})$ and germs of disjoint arcs that are labeled by objects of $\mathcal{C}$. The finitedimensional vector spaces $\mathcal{H}(\mathrm{E})=t_{\mathrm{ft}}(\mathrm{E})$ are called spaces of conformal blocks. As we will see, these vector spaces are expected to capture important properties of the (sheaves of) conformal blocks constructed from vertex algebras.

The morphisms of $\mathrm{Cob}_{\mathcal{C}}$ are cobordisms $\left(\mathrm{M}, \partial_{-} \mathrm{M}, \partial_{+} \mathrm{M}\right)$ containing a ribbon graph ("Wilson lines", in physicists' language) which is required to end at the germs of arcs on the boundary $\partial \mathrm{M}=\partial_{-} \mathrm{M} \sqcup \partial_{+} \mathrm{M}$. Segments of the ribbon graph are labeled by objects of $\mathcal{C}$, and vertices by suitable morphisms of $\mathcal{C}$. It follows immediately from the properties of a monoidal functor that for a cobordism of the form $(\mathrm{M}, \emptyset, \partial \mathrm{M})$ the functor $\mathrm{tft}_{\mathcal{C}}$ gives a linear map

$$
\operatorname{tft}_{\mathcal{C}}(\mathrm{M}) \equiv t t_{\mathcal{C}}(\mathrm{M}, \emptyset, \partial \mathrm{M}): \quad \mathbb{C} \rightarrow \mathcal{H}(\partial \mathrm{M}) .
$$

Thus this type of cobordism allows us to specify vectors in the space of conformal blocks associated to the boundary of a three-manifold with embedded ribbon graph.

Apart from monoidality, the functor $\mathrm{tft}_{\mathcal{C}}$ must obey a few more axioms (see e.g. $\left[\mathbf{F}^{3} \mathbf{S}\right.$ for the explicit statement) - naturality, multiplicativity, normalization, and functoriality, i.e. compatibility with gluing: if the cobordism $M$ is obtained by gluing two cobordisms $\mathrm{M}_{1}, \mathrm{M}_{2}$ with a morphism

$$
f: \quad \partial_{+} \mathrm{M}_{1} \rightarrow \partial_{-} \mathrm{M}_{2}
$$

of extended surfaces, then the corresponding linear maps $t f t_{\mathcal{C}}(\cdot)$ are related by

$$
\operatorname{tft}_{\mathcal{C}}(\mathrm{M})=\kappa^{m} t f t_{\mathcal{C}}\left(\mathrm{M}_{2}\right) \circ f_{\sharp} \circ \mathrm{tft}_{\mathcal{C}}\left(\mathrm{M}_{1}\right)
$$

with some complex number $\kappa$ that depends only on $\mathcal{C}$ and an integer $m$ that depends on the cobordisms $\mathrm{M}_{1}$ and $\mathrm{M}_{2}$, and on $f$. The functor $t f t t_{\mathcal{C}}$ thus provides a threedimensional topological field theory [Tu.

The axioms for $\mathrm{ft}_{\mathcal{C}}$ have two important consequences:

- They endow $\mathcal{H}(\mathrm{E})$ with a projective representation of the mapping class group Map(E) of E. 
- Taking a cylinder $\mathrm{E} \times[0,1]$ over an extended surface $\mathrm{E}$ and gluing two of its arcs by a tube on one side of the resulting cobordism allows one to obtain factorization isomorphisms for conformal blocks.

The term "conformal block" is also used in a mathematically a priori different context. Given a conformal vertex algebra $\mathcal{V}$ and an $m$-tuple $\left(\lambda_{1}, \lambda_{2}, \ldots, \lambda_{m}\right)$ of $\mathcal{V}$-modules, one can construct a vector bundle $\mathcal{B}\left(\lambda_{1}, \lambda_{2}, \ldots, \lambda_{m}\right)$ over the moduli space $\mathcal{M}_{g, m}$ of complex curves of genus $g$ with $m$ marked points. The action of the Virasoro algebra on these representations leads to a projectively flat connection on $\mathcal{B}$, the Knizhnik-Zamolodchikov connection. On the other hand, the structural morphisms that, for a sufficiently nice subclass of vertex algebras [Hu2, endow the representation category $\operatorname{Rep}(\mathcal{V})$ with the structure of a modular tensor category are deduced solely from monodromy data of conformal blocks on $\mathbb{C P}^{1}$.

Thus a rational vertex algebra, i.e. a vertex algebra whose representation category is a modular tensor category $\mathcal{C}$, supplies us with two a priori unrelated representations of the mapping class group $\pi_{1}\left(\mathcal{M}_{g, m}\right)$ : the one obtained from the monodromies of the vector bundles $\mathcal{B}$ over $\mathcal{M}_{g, m}$, and the one provided by the functor $\mathrm{tft}_{\mathcal{C}}$. We will, from now on, assume that these two representations coincide for all genera $g$ and all values of $m$. This assumption implies in particular an explicit formula for the rank of the vector bundles $\mathcal{B}\left(\lambda_{1}, \lambda_{2}, \ldots, \lambda_{m}\right)$, the Verlinde formula, which is of interest to algebraic geometers (see [So for a review). Factorization rules of conformal blocks provide constraints on the representations of mapping class groups. Our assumption is thus closely linked to factorization rules; the latter have been established for specific classes of models, see e.g. [TUY, FB].

The horizontal sections of $\mathcal{B}$ are, in general, multivalued. Therefore their relation to correlation functions of a conformal field theory calls for a clarification. Our assumptions about the monodromies of $\mathcal{B}$ allow us to address this question using the functor $t \mathrm{ft}_{\mathcal{C}}$.

\section{Some geometry for local conformal field theories}

Concretely, the desired clarification is provided by the following geometric construction. We are interested in a local CFT on a two-dimensional manifold X that can have a boundary. One must actually carefully distinguish between two different types of local CFTs: one in which the surface $\mathrm{X}$ is required to be oriented, and one in which no orientation of $\mathrm{X}$ is presupposed and accordingly also unorientable surfaces are admitted. However, for our purposes these two types of local CFTs can be treated simultaneously.

The first step is to replace $\mathrm{X}$ by the pair $(\widehat{\mathrm{X}}, \sigma)$ consisting of the complex double $\widehat{\mathrm{X}}$ of $\mathrm{X}$ and an orientation reversing involution $\sigma$. Notice that $\mathrm{X}$ can be recovered as the quotient by the action of the group $\langle\sigma\rangle$ : there is a projection

$$
\pi: \quad \widehat{\mathrm{X}} \mapsto \mathrm{X} \cong \widehat{\mathrm{X}} /\langle\sigma\rangle \text {. }
$$

We can now state the 'principle of holomorphic factorization' which relates local CFT on X to chiral CFT on $\widehat{\mathrm{X}}$ (for certain classes of conformal field theories this principle can formally be derived from an action functional $\mathbf{W i}$ ): a correlator $\operatorname{Cor}(\mathrm{X})$ on $\mathrm{X}$ is a specific vector in $\mathcal{H}(\widehat{\mathrm{X}})$. These vectors must obey two additional axioms: 
- They must be invariant under the action of $\operatorname{Map}(\mathrm{X}) \cong \operatorname{Map}(\widehat{\mathrm{X}})^{\sigma}$.

This group, also called the relative modular group $\mathbf{B S}$, acts genuinely on $\mathcal{H}(\widehat{\mathrm{X}})$, rather than only projectively.

- They must possess certain factorization properties.

We refer to FjFRS1, FjFRS2 for a precise formulation of these constraints.

In order to apply the relation to TFT outlined in section 1, we restrict our attention to rational full conformal field theories (RCFTs), that is, full CFTs whose correlators are obtained from the conformal blocks of a rational vertex algebra.

To put holomorphic factorization to work we now look for a cobordism $\left(\mathrm{M}_{\mathrm{X}}, \emptyset, \widehat{\mathrm{X}}\right)$ such that the vector $\operatorname{tft}_{\mathcal{C}}\left(\mathrm{M}_{\mathrm{X}}, \emptyset, \widehat{\mathrm{X}}\right) 1 \in \mathcal{H}(\widehat{\mathrm{X}})$ is the correlator $\operatorname{Cor}(\mathrm{X})$. It turns out that the following quotient of the interval bundle on $\widehat{\mathrm{X}}$ is appropriate:

$$
\mathrm{M}_{\mathrm{X}}=(\widehat{\mathrm{X}} \times[-1,1]) /\langle(\sigma, t \mapsto-t)\rangle .
$$

This three-manifold is oriented, has boundary $\partial \mathrm{M}_{\mathrm{X}} \cong \widehat{\mathrm{X}}$, and it contains $\mathrm{X}$ as a retract: the embedding 1 of $\mathrm{X}$ is to the fiber $t=0$, the retracting map contracts along the intervals.

The details of the construction are summarized in appendix A of FjFRS1. Here we content ourselves with emphasizing that it involves placing a uni-trivalent ribbon graph $\Gamma_{\mathrm{X}}$ on the union of $1(\mathrm{X})$ with the intervals over boundary points of $1(\mathrm{X})$. This graph contains in particular $1(\partial \mathrm{X})$. Univalent vertices of $\Gamma_{\mathrm{X}}$ are required to lie on $\pi^{-1}(\partial \mathrm{X}) \subset \widehat{\mathrm{X}} \cong \partial \mathrm{M}_{\mathrm{X}}$.

\section{Frobenius algebras}

The labeling of the ribbon graph $\Gamma_{\mathrm{X}}$ requires further data. The central idea of the TFT approach to RCFT correlators, as developed in [FRS1, FRS2, FRS4, FjFRS1 is that these data are provided by a (symmetric special) Frobenius algebra $A$ in the tensor category $\mathcal{C}$.

A Frobenius algebra $A$ in $\mathcal{C}$ is, by definition, an object of $\mathcal{C}$ carrying the structures of a unital associative algebra and of a counital coassociative coalgebra in $\mathcal{C}$, satisfying the compatibility requirement that the coproduct $\Delta: A \rightarrow A \otimes A$ is a morphism of $A$-bimodules (or, equivalently, that the product $m: A \otimes A \rightarrow A$ is a morphism of $A$-bi-comodules). A Frobenius algebra is called special iff the coproduct is a right-inverse to the product - this means in particular that the algebra is separable - and a multiple of the unit is a right-inverse to the counit. $A$ is called symmetric iff the two isomorphisms $A \rightarrow A^{\vee}$ that are naturally induced by product, counit and duality coincide.

We mention that in case $\mathrm{X}$ is unoriented, still more structure is needed: $A$ must then be a Jandl algebra, i.e. a symmetric special Frobenius algebra coming with an algebra isomorphism $A \rightarrow A^{\text {opp }}$ that squares to the twist $\theta_{A}$. This turns out to be the appropriate generalization of the notion of an algebra with involution to braided tensor categories.

Given such a symmetric special Frobenius algebra $A$, one can identify boundary conditions of the CFT with $A$-modules. Boundary fields $\Psi_{U}^{M N}$ that change the boundary condition from $M$ to $N$ and have chiral label $U$ are in bijection with $A$-module morphisms in $\operatorname{Hom}_{A}(M \otimes U, N)$. Types of (topological) defect lines can be identified with isomorphism classes of $A$-bimodules. The disorder fields $\Psi_{U V}^{B_{1} B_{2}}$ which change a defect line from type $B_{1}$ to type $B_{2}$ carry two chiral labels $U$ and $V$. 
They are in bijection with $A$-bimodule morphisms in $\operatorname{Hom}_{A \mid A}\left(U \otimes^{+} B_{1} \otimes^{-} V, B_{2}\right)$. Here the left and right $A$-actions $\rho_{l / r}$ on the bimodule $B_{1}$ are used to define a bimodule structure on the object $U \otimes B_{1} \otimes V$ by $\left(\operatorname{id}_{U} \otimes \rho_{l} \otimes \mathrm{id}_{V}\right) \circ\left(c_{U, A}^{-1} \otimes \mathrm{id}_{B_{1}} \otimes \mathrm{id}_{V}\right)$ and $\left(\operatorname{id}_{U} \otimes \rho_{r} \otimes \operatorname{id}_{V}\right) \circ\left(\operatorname{id}_{U} \otimes \operatorname{id}_{B_{1}} \otimes c_{A, V}^{-1}\right)$, respectively.

Notice that $A$ itself has a canonical structure of an $A$-bimodule; in fact, it is the tensor unit in the tensor category $\mathcal{C}_{A \mid A}$ of $A$-bimodules. In applications to conformal field theory, $A$ is required to be simple as a bimodule over itself. In the CFT context, we also call $A$ the invisible defect. Bulk fields of the CFT are the special disorder fields that correspond to $B_{1}=B_{2}=A$. As a consequence, the degeneracy of bulk fields with chiral labels $U_{i}$ and $U_{j}$ is given by

$$
Z_{i j}:=\operatorname{dim}_{\mathbb{C}} \operatorname{Hom}_{A \mid A}\left(U_{i} \otimes^{+} A \otimes^{-} U_{j}, A\right) .
$$

Thus such expressions yield modular invariant bulk field partition functions of CFTs, a result that has been obtained earlier BEK in the framework of subfactor theory.

The Frobenius algebra $A$ is, of course, also a module over itself and thus corresponds to a boundary condition. But in fact any other $A$-module $M$ supplies us with a symmetric special Frobenius algebra as well, namely the internal End End $(M)$. All these algebras $\underline{\operatorname{End}}(M)$ are Morita equivalent to $A$, and in the TFT approach they all provide equivalent descriptions of a full local CFT.

On the basis of these informations one can establish, for instance, the following further results:

I One can derive concrete expressions for partition functions of boundary, bulk and defect fields. Their coefficients are non-negative integers, and they satisfy consistency requirements like being modular invariant and forming NIMreps of the fusion rules [FRS1.

(However, not every bilinear combination of characters that is invariant under the action of the modular group is indeed the partition function of a conformal field theory. Many counterexamples are known.)

II These results can be extended to unorientable surfaces, for which the Frobenius algebra must be a Jandl algebra [FRS2, FRS5.

III The expressions for correlation functions can be made particularly explicit FRS3 for theories with torus partition function of simple current type; some details will be given below.

It is worth stressing, though, that the TFT approach to RCFT correlators treats all RCFTs - the simple current case and those with exceptional modular invariants - on an equal footing.

IV One can derive explicit expressions for the coefficients of operator product expansions [FRS4].

V Finally, one can prove FjFRS1 modular invariance, at arbitrary genus, and factorization of the correlators obtained in the TFT approach.

\section{Picard groups}

From the discussion above we learn in particular that in any full local conformal field theory two tensor categories are encountered naturally: the (modular) tensor category $\mathcal{C}$ that describes the chiral data, and the tensor category $\mathcal{C}_{A \mid A}$ of $A$-bimodules that describes defects and their fusion. By standard arguments, the 
latter does not depend on the choice of $A$ within one and the same Morita class. It should be noted that $\mathcal{C}_{A \mid A}$ is not, in general, a braided tensor category. This fits well with the absence of any physically reasonable notion of a braiding of defects. The following construction can be applied to both tensor categories $\mathcal{C}$ and $\mathcal{C}_{A \mid A}$.

An object $V$ of a tensor category with simple tensor unit is called invertible iff there exists an object $W$ such that $V \otimes W \cong \mathbf{1} \cong W \otimes V$. The tensor product of $\mathcal{C}$ endows the set of isomorphism classes of invertible objects with the structure of a group, the Picard group $\operatorname{Pic}(\mathcal{C}) \subset K_{0}(\mathcal{C})$. The Picard group of a braided tensor category is abelian. If $\mathcal{C}$ is the representation category of a rational vertex algebra, the term 'simple currents' $[\mathbf{S Y}]$ is also used for elements of the Picard group.

The following two results allow one to control aspects of tensor categories, given their Picard groups:

- Let $G$ be a finite group. Monoidal categories $\mathcal{C}$ that are enriched over $\mathcal{V e c t}_{\mathbb{C}}$ together with an isomorphism $K_{0}(\mathcal{C}) \rightarrow \mathbb{Z} G$ are in bijection with elements of the cohomology group $H^{3}\left(G, \mathbb{C}^{\times}\right)$.

- Let $G$ be a finite abelian group. Braided monoidal categories $\mathcal{C}$ enriched over $\mathcal{V} e c t_{\mathbb{C}}$ together with an isomorphism $K_{0}(\mathcal{C}) \rightarrow \mathbb{Z} G$ are in in bijection with elements of the group $H_{\mathrm{ab}}^{3}\left(G, \mathbb{C}^{\times}\right)$of Eilenberg-Mac Lane's [EM] abelian group cohomology.

It turns out that elements of $H_{\mathrm{ab}}^{3}\left(G, \mathbb{C}^{\times}\right)$are in bijection with quadratic forms on $G$. In the case at hand, the relevant quadratic form on the Picard group is given by the eigenvalue of the twist, i.e. by (the exponential of minus $2 \pi \mathrm{i}$ times) the fractional part of the conformal weight.

It is now time to present some examples for symmetric special Frobenius algebras. The tensor unit is such an algebra and, more generally, for any object $U$ of $\mathcal{C}$ with $\operatorname{dim}(U) \neq 0$ the object $U \otimes U^{\vee}$ can be endowed with such an algebra structure. These algebras are all Morita equivalent; they yield in (1) the (charge-) diagonal bulk field partition function, $Z_{i j}=\delta_{i, j^{\vee}}$. This situation has been termed the "Cardy case" in the physics literature.

The next tractable class of symmetric special Frobenius algebras are Schellekens algebras. A Schellekens algebra is a symmetric special Frobenius algebra for which each simple subobject is invertible and which is simple as a left-module over itself. Every such algebra can be constructed from a subgroup $H \leq \operatorname{Pic}(\mathcal{C})$ (to be called the support of $A$ ) and a cochain $\omega: H \times H \rightarrow \mathbb{C}^{\times}$with $\mathrm{d} \omega=\left.\psi\right|_{H}$, where $\psi$ represents the class in $H^{3}\left(G, \mathbb{C}^{\times}\right)$that determines the monoidal structure of the Picard subcategory of $\mathcal{C}$, i.e. of the full tensor subcategory of $\mathcal{C}$ generated by the invertible objects. For any symmetric special Frobenius algebra that is simple as a left module over itself, one has the estimate Ga, FRS3

$$
\operatorname{dim}_{\mathbb{C}} \operatorname{Hom}(U, A) \leq \operatorname{dim}(U)
$$

as a consequence, the object underlying a Schellekens algebra is a direct sum $\bigoplus_{h \in H} U_{h}$ of pairwise non-isomorphic invertible objects.

It is, in fact, appropriate to regard Schellekens algebras as the generalization to braided categories of twisted group algebras. The latter are classified by $H^{2}\left(G, \mathbb{C}^{\times}\right)$; for an abelian group $G$, this cohomology group is isomorphic to the 
group $A B\left(G, \mathbb{C}^{\times}\right)$of alternating bicharacters of $G$, an isomorphism being given by

$$
\begin{aligned}
H^{2}\left(G, \mathbb{C}^{\times}\right) & \rightarrow A B\left(G, \mathbb{C}^{\times}\right) \\
{[\omega] } & \mapsto \xi(g, h):=\frac{\omega(g, h)}{\omega(h, g)} .
\end{aligned}
$$

While an alternating bicharacter $\xi$ is characterized by $\xi(g, g)=1$, its appropriate braided generalization, a Kreuzer-Schellekens bihomomorphism (KSB), is a bicharacter $\Xi$ whose diagonal values are given by the eigenvalues of the twist on the invertible objects, $\Xi(g, g)=\theta_{g}$.

Next we realize that the multiplication on a Schellekens algebra provides us with a KSB. Indeed, for a Schellekens algebra of support $H \leq \operatorname{Pic}(\mathcal{C})$, pick for each group element $\left[U_{g}\right] \in H$ a nonzero morphism $\iota_{g} \in \operatorname{Hom}\left(U_{g}, A\right)$. The space $\operatorname{Hom}\left(U_{g} \otimes U_{h}, U_{g h}\right)$ is one-dimensional, and hence there exist numbers $\Xi_{A}(h, g)$ such that

$$
m \circ c_{A, A} \circ\left(\iota_{g} \otimes \iota_{h}\right)=\Xi_{A}(h, g) m \circ\left(\iota_{g} \otimes \iota_{h}\right) .
$$

It should be appreciated that the resulting function

$$
\Xi_{A}: \quad H \times H \rightarrow \mathbb{C}
$$

does not depend on the choice of the morphisms $\iota_{g}$, but depends on the multiplication on $A$. One shows that $\Xi_{A}(h, g)$ is a KSB, and that a Schellekens algebra is uniquely characterized, up to isomorphism, by the support $H$ of $A$ and the choice of a KSB on $H$.

We are now in a position to express quantities of interest in conformal field theory in terms of the KSB. First, we obtain the partition function

$$
Z_{i j}(A)=\frac{1}{|H(A)|} \sum_{g, h \in H(A)} \vartheta_{i}(h) \Xi_{A}(h, g) \delta_{j^{\vee}, g i}
$$

for bulk fields, which reproduces a result of $\left[\mathbf{K S}\right.$. Here $\vartheta_{i}(h)$ is the so-called (exponentiated) monodromy charge of $U_{i}$ with respect to $h$, which is the combination

$$
\vartheta_{i}(h):=\theta_{h i} \theta_{h}^{-1} \theta_{i}^{-1}
$$

of twist eigenvalues.

Boundary conditions can be determined from the decomposition of induced $A$ modules into simple $A$-modules. They correspond to orbits of the support $H(A)$ on (isomorphism classes of) simple objects of $\mathcal{C}$ and representations of the twisted group algebra $\mathbb{C}_{\epsilon_{U}} \mathcal{S}_{U}$, where $\mathcal{S}_{U} \leq H(A)$ is the stabilizer of the simple object $U$ and the alternating bicharacter $\epsilon_{U}$ on $\mathcal{S}_{U}$ is given by

$$
\epsilon_{U}(g, h)=\phi_{U}(g, h) \Xi_{A}(h, g),
$$

i.e. is the product of the restriction of the KSB $\Xi_{A}$ to $\mathcal{S}_{U}$ and a certain two-cochain $\phi_{U}$. The latter is a gauge independent $6 j$-symbol, defined by

$$
\gamma_{g} \circ\left(\operatorname{id}_{U_{g}} \otimes \delta_{h}\right)=: \phi_{U}(g, h) \delta_{h} \circ\left(\gamma_{g} \otimes \operatorname{id}_{U_{h}}\right),
$$

with $\left\{\gamma_{g}\right\}$ and $\left\{\delta_{h}\right\}$ (arbitrary) bases of the one-dimensional morphism spaces $\operatorname{Hom}\left(U_{g} \otimes U, U\right)$ and $\operatorname{Hom}\left(U \otimes U_{h}, U\right)$, respectively. A similar description exists for defect lines. Also, explicit formulae for boundary states have been derived in [FRS3, which prove conjectures made in [FHSSW]. 


\section{Twining characters}

The gauge independent $6 j$-symbols $\phi_{U}$ defined in formula (3) deserve further study. One can show that, for a general modular tensor category, they are bicharacters, in fact KSBs, on the stabilizer $\mathcal{S}_{U}$. To proceed, consider the special case of chiral WZW conformal field theories: As is well known, to each pair $(\mathfrak{g}, k)$ with $\mathfrak{g}$ a finite-dimensional complex simple Lie algebra and $k \in \mathbb{N}$, one can associate a modular tensor category $\mathcal{C}(\mathfrak{g}, k)$. It can be realized as the category of integrable highest weight modules at level $k$ of the untwisted affine Lie algebra $\hat{\mathfrak{g}}$ with horizontal subalgebra $\mathfrak{g}$, or as the representation category of the vertex algebra $\mathcal{V}(\mathfrak{g}, k)$ that can be defined on the irreducible highest weight $\mathfrak{g}$-module with highest weight $k \Lambda_{(0)}$.

For these categories, conformal field theory leads to a conjecture, to be presented as relation (6) below, that links the $6 j$-symbols $\phi_{U}(g, h)$ to representation theoretic quantities, the so-called twining characters FSS1, FRaS (or graded traces, see [DLM]). A symmetry $\dot{\omega}$ of the Dynkin diagram of a Kac-Moody algebra (or generalized Kac-Moody algebra) $\hat{\mathfrak{g}}$ gives rise to an outer automorphism $\omega$ of $\hat{\mathfrak{g}}$ that respects a triangular decomposition. We call such an automorphism of $\hat{\mathfrak{g}}$ a diagram automorphism. It should be noted that in the case that $\hat{\mathfrak{g}}$ is an untwisted affine Lie algebra and $\dot{\omega}$ acts nontrivially on the node corresponding to the vacuum representation, these Lie algebra automorphisms do not give rise to automorphisms of the vertex algebra $\mathcal{V}(\mathfrak{g}, k)$.

Now recall that the simple objects of $\mathcal{C}(\mathfrak{g}, k)$ are irreducible highest weight representations of the untwisted affine Lie algebra $\hat{\mathfrak{g}}$. As for any tensor category, the Picard group of $\mathcal{C}(\mathfrak{g}, k)$ acts on the isomorphism classes of simple objects by the tensor product. Since an invertible object $L$ obeys $L \otimes L^{\vee} \cong \mathbf{1}$ and since $\operatorname{dim}(L)=\operatorname{dim}\left(L^{\vee}\right)$, the (quantum) dimension of $L$ fulfils $(\operatorname{dim}(L))^{2}=1$. When scanning integrable $\hat{\mathfrak{g}}$-modules with this property one finds $[\mathbf{F u}]$ that for any pair $(\mathfrak{g}, k)$ (with the exception of $\left(E_{8}, 2\right)$, which is to be excluded from the discussion below), every element of the Picard group corresponds to a diagram automorphism of $\hat{\mathfrak{g}}$.

Given an automorphism $\varpi$ of $\hat{\mathfrak{g}}$ that preserves a fixed Borel subalgebra and an irreducible highest weight representation $(V, \rho)$ with $\rho: \hat{\mathfrak{g}} \rightarrow \mathfrak{g l}(V)$, another irreducible highest weight representation $\left(V, \rho^{\varpi}\right)$ is obtained by setting $\rho^{\varpi}:=\rho \circ \varpi$. If $\varpi=\omega$ is a diagram automorphism, then the highest weight spaces of these two representations coincide. If the irreducible representations $(V, \rho)$ and $\left(V, \rho^{\omega}\right)$ are isomorphic, the representation is called a fixed point of the automorphism $\omega$. In this case, there exists a unique linear map $\mathcal{T}_{\omega}: V \rightarrow V$ that restricts to the identity on the highest weight space and obeys $\rho \circ \omega(x) \mathcal{T}_{\omega}=\mathcal{T}_{\omega} \rho(x)$ for all $x \in \hat{\mathfrak{g}}$. Given an irreducible highest weight representation $\left(V_{\Lambda}, \rho_{\Lambda}\right)$ of highest weight $\Lambda$ that is a fixed point for some diagram automorphism $\omega$, one defines the (Virasoro-specialized) twining character of $\left(V_{\Lambda}, \rho_{\Lambda}\right)$ as the function

$$
\chi_{\Lambda}^{\omega}(\tau):=\operatorname{Tr}_{V_{\Lambda}} \mathcal{T}_{\omega} \exp \left(2 \pi \mathrm{i} \tau\left(L_{0}-\frac{c}{24}\right)\right)
$$

on the complex upper half plane, where $L_{0}$ is the action of the zero mode of the Virasoro algebra obtained by the Sugawara construction.

In particular, given an element $g$ of the Picard group $\operatorname{Pic}(\mathcal{C}(\mathfrak{g}, k))$, we obtain a twining character for each integrable highest $\Lambda$ that is a fixed point under the corresponding Lie algebra automorphism $\omega_{g}$. Explicit character formulae for 
the twining characters of irreducible highest weight modules have been derived in FSS1, FRaS. (For twining characters of other types, see [KK, We, Na1, Na2 .) From these character formulae, it follows that, under the usual action of the modular group $S L(2, \mathbb{Z})$ on the upper half plane, these twining characters transform into linear combinations of twining characters for the same automorphism $\omega_{g}-\mathrm{a}$ non-trivial empirical observation from the point of view of Lie theory. In particular one finds

$$
\chi_{\Lambda}^{\omega_{g}}\left(-\frac{1}{\tau}\right)=\sum_{\Lambda^{\prime}} S_{\Lambda, \Lambda^{\prime}}^{\omega_{g}} \chi_{\Lambda^{\prime}}^{\omega_{g}}(\tau)
$$

with a unitary and symmetric matrix $S^{\omega_{g}}$.

The following conjecture relates the matrix $S^{\omega_{g}}$ obtained this way to the gauge independent $6 j$-symbols $\phi_{U}$ defined by (3):

$$
S_{\Lambda, h \Lambda^{\prime}}^{\omega_{g}}=\vartheta_{\Lambda}(h) \phi_{\Lambda^{\prime}}(g, h)^{-1} S_{\Lambda, \Lambda^{\prime}}^{\omega_{g}},
$$

where $\vartheta_{\Lambda}(g)=\theta_{g \Lambda} \theta_{g}^{-1} \theta_{\Lambda}^{-1}$ is the exponentiated monodromy charge, which we already encountered in (2).

A proof of this conjecture seems to be out of reach at present. However, the formula has been checked in a huge number of non-trivial cases; most of these checks have been performed with a sophisticated computer package $\mathbf{k a c}$. For the role of the $6 j$-symbols played in the untwisted stabilizer, we also refer to Ba1, Ba2. The matrices $S^{\omega_{g}}$ are also an important ingredient in refinements of the Verlinde conjecture for non-simply connected Lie groups [FSS2, [FS].

\section{The bimodule Picard group}

Let us now turn our attention to the Picard group of the category $\mathcal{C}_{A \mid A}$ of $A$-bimodules. This group is of considerable interest, too: it turns out [FFRS1 that it describes internal symmetries of a local CFT. Since the tensor category $\mathcal{C}_{A \mid A}$ is, in general, not braided, this group of symmetries can be non-abelian. To give some examples, for the Ising model one has $\operatorname{Pic}\left(\mathcal{C}_{A \mid A}\right) \cong \operatorname{Pic}(\mathcal{C}) \cong \mathbb{Z}_{2}$, while for the critical three-state Potts model we find a non-abelian symmetry group, $\operatorname{Pic}\left(\mathcal{C}_{A \mid A}\right) \cong S_{3}$. These results conincide with the symmetries of the Ising and the three-state Potts model on a lattice which, as their names suggest, produce the corresponding conformal field theories in the continuum limit.

The action of an invertible bimodule $B$ on a boundary condition $M$ of the CFT is given by

$$
M \mapsto B \otimes_{A} M,
$$

where $\otimes_{A}$ is the tensor product over $A$. For the action on boundary fields, we find

$$
\operatorname{Hom}\left(M_{1} \otimes U, M_{2}\right) \mapsto \operatorname{Hom}\left(\left(B \otimes_{A} M_{1}\right) \otimes U, B \otimes_{A} M_{2}\right) .
$$

For the action on bulk fields, which is somewhat more involved, we refer to FFRS1, FFRS2.

If $A=A\left(H, \Xi_{A}\right)$ is a Schellekens algebra, then there is a subgroup of internal symmetries of the form

$$
H^{*} \times{ }_{H} \operatorname{Pic}(\mathcal{C}),
$$

where $H$ is mapped to its character group $H^{*}$ by the homomorphism $H \rightarrow H^{*}$, $H \ni h \mapsto \Xi_{A}(\cdot, h)$ and then acts by right-multiplication. This statement can be derived as a special case of the following result: Suppose that $\mathcal{C}^{\prime}$ is a braided tensor 
(in fact, semisimple ribbon) category with the property that $\operatorname{Pic}\left(\mathcal{C}^{\prime}\right)$ acts freely on the isomorphism classes of simple objects of $\mathcal{C}^{\prime}$. (For any tensor category, the full subcategory of all invertible objects has this property.) Let $A$ be a Schellekens algebra in $\mathcal{C}^{\prime}$ with support $H$. Then the fusion ring of $A$-bimodules is given by

$$
K_{0}\left(\mathcal{C}_{A \mid A}^{\prime}\right) \cong K_{0}\left(\mathcal{C}^{\prime}\right) \otimes_{\mathbb{Z} H} \mathbb{Z} H^{*} .
$$

Here the group ring $\mathbb{Z} H$ is regarded as a subring of $K_{0}\left(\mathcal{C}^{\prime}\right)$, and its action on $\mathbb{Z} H^{*}$ is again defined using the $\mathrm{KSB} \Xi_{A}$ of $A$.

The result (7) can be seen as follows (for details see [FFRS3). One first shows that the automorphism group of a Schellekens algebra $A=\bigoplus_{h \in H} U_{h}$ is canonically isomorphic to the character group $H^{*}$. This extends results about the relationship between simple current extensions and orbifolds by abelian groups. Next one shows that every simple $A$-bimodule is isomorphic to a bimodule of the form $A \otimes U$ with left action $m \otimes \mathrm{id}_{U}$ and right action $\left(m \otimes \mathrm{id}_{U}\right) \circ\left(\mathrm{id}_{A} \otimes c_{U A}\right) \circ\left(\mathrm{id}_{A \otimes U} \otimes \psi\right)$ for some algebra automorphism $\psi$ of $A$. The tensor product over $\mathbb{Z} H$ in (7) takes care of an over-parametrization in a way that is compatible with the fusion rules.

These considerations can be generalized to include not only internal symmetries, but also duality symmetries of Kramers-Wannier type. The fact that $A$ is a Frobenius algebra implies that the monoidal category $\mathcal{C}_{A \mid A}$ comes with a duality; it is even a sovereign tensor category. The conformal field theory has KramersWannier like dualities whenever there is an object $B \in \mathcal{C}_{A \mid A}$ such that $B \otimes_{A} B^{\vee}$ is a direct sum of invertible bimodules, i.e. is in the Picard subcategory of $\mathcal{C}_{A \mid A}$. The well-known Kramers-Wannier duality for the Ising model can thus be deduced from the fusion rule $\sigma \otimes \sigma \cong \mathbf{1} \oplus \epsilon$ for the primary field $\sigma$ of conformal weight $\frac{1}{16}$, in agreement with another approach to such dualities based on the symmetries of boundary states $[\mathbf{R u}$.

\section{Conclusions}

The TFT approach to the construction of CFT correlators provides a powerful algebraization of many questions arising in conformal field theory. This allows both for making rigorous statements about rational conformal field theories and for setting up efficient algorithms. A rich dictionary that relates algebraic concepts and physical notions is emerging; the relation between Picard groups and symmetry groups is merely one example.

Interesting algebraic structures also show up when a route analogous to the TFT approach is taken in the framework of vertex algebras. The results of HK1, HK2 should be seen as just the beginning of a rich extension of the theory of vertex algebras.

To summarize, a central idea of the construction presented in this article is to represent conformal field theory quantities as invariants of knots and links in three-manifolds. Tools for achieving this are provided by topological field theory, which in turn is based on the notion of a modular tensor category. It seems thus fair to say that the tensor structure present for the modules over the underlying vertex algebra, which is due in particular to the work of Jim Lepowsky, is a key ingredient in the construction. 


\section{References}

[Ba1] P. Bantay, The untwisted stabilizer in simple current extensions, Phys. Lett. B 396 (1997), 183-185 [hep-th/9611194]

[Ba2] Simple current extensions and mapping class group representations, Int. J. Mod. Phys. A 13 (1998), 199-208 [hep-th/9611124]

[BS] M. Bianchi and A. Sagnotti, Open strings and the relative modular group, Phys. Lett. B 231 (1989), 389-396

[BEK] J. Böckenhauer, D.E. Evans, and Y. Kawahigashi, Longo-Rehren subfactors arising from $\alpha$-induction, Publ. RIMS 37 (2001), 1-35 [math.0A/0002154]

[DLM] C. Dong, H. Li, and G. Mason, Modular-invariance of trace functions in orbifold theory and generalized moonshine, Commun. Math. Phys. 214 (2000), 1-56 [q-alg/9703016]

[EM] S. Eilenberg and S. MacLane, Cohomology theory of abelian groups and homotopy theory II, Proc. Natl. Acad. Sci. USA 36 (1950), 657-663

$\left[\mathrm{F}^{3} \mathrm{~S}\right] \quad$ G. Felder, J. Fröhlich, J. Fuchs, and C. Schweigert, Correlation functions and boundary conditions in RCFT and three-dimensional topology, Compos. Math. 131 (2002), 189237 [hep-th/9912239]

[FjFRS1] J. Fjelstad, J. Fuchs, I. Runkel, and C. Schweigert, TFT construction of RCFT correlators V: Proof of modular invariance and factorisation, preprint hep-th/0503194

[FjFRS2] _ Topological and conformal field theory as Frobenius algebras, preprint math. CT/0512076

[FB] E. Frenkel and D. Ben-Zvi, Vertex Algebras and Algebraic Curves, 2nd edition (American Mathematical Society, Providence 2004)

[FFRS1] J. Fröhlich, J. Fuchs, I. Runkel, and C. Schweigert, Kramers-Wannier duality from conformal defects, Phys. Rev. Lett. 93 (2004), 070601 [cond-mat/0404051]

[FFRS2] , Picard groups in rational conformal field theory, Contemp. Math. 391 (2005), 85-100 [math.CT/0411507]

[FFRS3] Duality and defects in rational conformal field theory, preprint in preparation

[Fu] J. Fuchs, Simple WZW currents, Commun. Math. Phys. 136 (1991), 345-356

[FHSSW] J. Fuchs, L.R. Huiszoon, A.N. Schellekens, C. Schweigert, and J. Walcher, Boundaries, crosscaps and simple currents, Phys. Lett. B 495 (2000), 427-434 [hep-th/0007174]

[FRaS] J. Fuchs, U. Ray, and C. Schweigert, Some automorphisms of Generalized Kac-Moody algebras, J. Algebra 191 (1997), 518-540 [q-alg/9605046]

[FRS1] J. Fuchs, I. Runkel, and C. Schweigert, TFT construction of RCFT correlators I: Partition functions, Nucl. Phys. B 646 (2002), 353-497 [hep-th/0204148]

[FRS2] - TFT construction of RCFT correlators II: Unoriented world sheets, Nucl. Phys. B 678 (2004), 511-637 [hep-th/0306164]

[FRS3] - TFT construction of RCFT correlators III: Simple currents, Nucl. Phys. B 694 (2004), 277-353 [hep-th/0403157]

[FRS4] , TFT construction of RCFT correlators IV: Structure constants and correlation functions, Nucl. Phys. B 715 (2005), 539-638 [hep-th/0412290]

[FRS5] , Ribbon categories and (unoriented) CFT: Frobenius algebras, automorphisms, reversions, preprint math. CT/0511590

[FSS1] J. Fuchs, A.N. Schellekens, and C. Schweigert, From Dynkin diagram symmetries to fixed point structures, Commun. Math. Phys. 180 (1996), 39-98 [hep-th/9506135]

[FSS2] _ A matrix S for all simple current extensions, Nucl. Phys. B 473 (1996), 323366 [hep-th/9601078]

[FS] J. Fuchs and C. Schweigert, The action of outer automorphisms on bundles of chiral blocks, Commun. Math. Phys. 206 (1999), 691-736 [hep-th/9805026]

[Ga] T. Gannon, Boundary conformal field theory and fusion ring representations, Nucl. Phys. B 627 (2002), 506-564 [hep-th/0106105]

[Hu1] Y.-Z. Huang, A theory of tensor products for module categories for a vertex operator algebra, IV, J. Pure Appl. Alg. 100 (1995), 173-216 [q-alg/9505019]

[Hu2] Vertex operator algebras, fusion rules and modular transformations, Contemp. Math. 391 (2005), 135-148 [math.QA/0502558]

[HK1] Y.-Z. Huang and L. Kong, Open-string vertex algebras, tensor categories and operads, Commun. Math. Phys. 250 (2004), 433-471 [math.QA/0308248]

[HK2] , Full field algebras, preprint math.QA/0511328 
[HL1] Y.-Z. Huang and J. Lepowsky, Tensor products of modules for a vertex operator algebra and vertex tensor categories, in: Lie Theory and Geometry, R. Brylinski et al., eds. (Birkhäuser, Boston 1994), p. 349-383 |hep-th/9401119|

[HL2] - A theory of tensor products for module categories for a vertex operator algebra, I, Selecta Mathematica 1 (1995), 699-756 [hep-th/9309076]

[HL3] A theory of tensor products for module categories for a vertex operator algebra, II, Selecta Mathematica 1 (1995), 757-786 [hep-th/9309159]

[HL4] A theory of tensor products for module categories for a vertex operator algebra, III, J. Pure Appl. Alg. 100 (1995), 141-171 [q-alg/9505018]

[kac] Computer program KAC, written by A.N. Schellekens; available at http://www.nikhef.nl/ t58/kac.html

[KK] S.-J. Kang and J.-H. Kwon, Graded Lie superalgebras, supertrace formula, and orbit Lie superalgebras, Proc. London Math. Soc. 81 (2000), 675-724

[KS] M. Kreuzer and A.N. Schellekens, Simple currents versus orbifolds with discrete torsion - a complete classification, Nucl. Phys. B 411 (1994), 97-121 [hep-th/9306145]

[MS] G. Moore and N. Seiberg, Classical and quantum conformal field theory, Commun. Math. Phys. 123 (1989), 177-254

[Na1] S. Naito, Twining character formula of Kac-Wakimoto type for affine Lie algebras, Represent. Theory 6 (2002), 70-100

[Na2] Twining character formula of Borel-Weil-Bott type, J. Math. Sci. Tokyo 9 (2002), 637-658

[RT] N.Yu. Reshetikhin and V.G. Turaev, Ribbon graphs and their invariants derived from quantum groups, Commun. Math. Phys. 127 (1990), 1-26

[Ru] Ph. Ruelle, Kramers-Wannier dualities via symmetries, Phys. Rev. Lett. 95 (2005), 225701 [cond-mat/0504758]

[SY] A.N. Schellekens and S. Yankielowicz, Simple currents, modular invariants, and fixed points, Int. J. Mod. Phys. A 5 (1990), 2903-2952

[So] Ch. Sorger, La formule de Verlinde, Astérisque 237 (1996), 87-114

[Tu] V.G. Turaev, Quantum Invariants of Knots and 3-Manifolds (de Gruyter, New York 1994)

[TUY] A. Tsuchiya, K. Ueno, and H. Yamada, Conformal field theory on universal family of stable curves with gauge symmetries, Adv. Studies in Pure Math. 19 (1989), 459-566

[We] R. Wendt, Weyl's character formula for non-connected Lie groupss and orbital theory for twisted affine affine Lie algebras, J. Funct. Anal. 180 (2001), 31-65 [math.RT/ 9909059]

[Wi] E. Witten, On holomorphic factorization of WZW and coset models, Commun. Math. Phys. 144 (1992), 189-212

Organisationseinheit Mathematik, Universität Hamburg, Bundesstraße 55, D - 20146 HAMBURG

E-mail address: schweigert@math.uni-hamburg.de

Avdelning fysik, Karlstads Universitet, Universitetsgatan 5, S-65188 Karlstad

E-mail address: jfuchs@fuchs.tekn.kau.se

Department of Mathematics, King's College London, Strand, London WC2R 2LS

E-mail address: ingo@mth.kcl.ac.uk 